\title{
Energetic Suppression of Decoherence in Exchange-Only Quantum Computation
}

\author{
Yaakov S. Weinstein ${ }^{1, *}$ and C. Stephen Hellberg ${ }^{1, \text { 由 }}$ \\ ${ }^{1}$ Center for Computational Materials Science, Naval Research Laboratory, Washington, DC 20375
}

\begin{abstract}
Universal quantum computation requiring only the Heisenberg exchange interaction and suppressing decoherence via an energy gap is presented. The combination of an always-on exchange interaction between the three physical qubits comprising the encoded qubit and a global magnetic field generates an energy gap between the subspace of interest and all other states. This energy gap suppresses decoherence. Always-on exchange couplings greatly simplify hardware specifications and the implementation of inter-logical-qubit gates. A controlled phase gate can be implemented using only three Heisenberg exchange operations all of which can be performed simultaneously.
\end{abstract}

PACS numbers: 03.67.Lx, 03.67.Pp, 75.10.Jm

Encoding logical qubits (LQ) into subspaces of systems with more then one physical qubit is a powerful protocol in quantum information processing. Subspaces of this kind have been shown to protect quantum information from decoherence due to interactions with the environment, while still allowing for universal quantum computation 1, 2]. Experimental examples of these decoherence free subspaces (DFS) have been realized on nuclear magnetic resonance [3], ion trap [4], and optical systems [5], have been suggested for superconducting qubits [6], and have been used to implement encoded quantum algorithms 7, 8]. Encoded subspaces known as interaction free subspaces (IFS) can be used to protect information from always-on inter-qubit couplings. This reduces hardware constraints in certain system by circumventing the need to turn on and off two-qubit interactions. Rather, when the interaction is desired, the information is simply taken out of IFS [9, 10]. A combination of DFS and IFS for universal computation has recently been proposed 11]. Finally, encoded subspaces allow performance of quantum logic maximizing the use of readily available operators while partially or completely avoiding operators that may add complexity to the computing hardware or a significant amount of time to the computation. Specifically, this type of subspace has been suggested to perform universal quantum computation with only the Heisenberg exchange interaction [12, 13, 14].

Many proposals for the implementation of quantum computation, including quantum dots [15] and other spin based methods 16, 17], rely on the Heisenberg exchange as the means of inter-qubit interactions. This is, in part, due to the many desirable qualities found in Heisenberg exchange: it is short ranged, allows fast gate operation, and has an on-off ratio of many orders of magnitude [18. However, without encoding, the Heisenberg ex-

${ }^{*}$ To whom correspondence should be addressed; Electronic address: weinstei@dave.nrl.navy.mil

${ }^{\dagger}$ Electronic address: hellberg@dave.nrl.navy.mil change alone is not universal. Experimental proposals of quantum computation thus supplement the Heisenberg exchange with one qubit gates which require local magnetic fields [15] or g-factor engineering [19]. This puts strenuous demands on the quantum computing hardware and may significantly reduce computational speed.

To circumvent the difficulty of one qubit gates in solidstate systems, methods have been devised to embed LQs in subspaces of more than one physical qubit in such a way that the exchange interaction becomes universal. One such encoding, introduced by DiVincenzo et al [12], calls for each LQ to be embedded in three physical qubits. Exchange interactions within the LQ can perform global rotations while operations between LQs can be used for two-qubit gates. A minimization search found that 19 such operations were needed to perform a CNOT gate.

A second encoding strategy to avoid single qubit rotations, suggested by Levy 13, 20, embeds the LQ into two physical qubits residing in inequivalent local environments. For example, if two quantum-dot qubits have different $g$-factors, a magnetic field will cause a phase difference between the physical qubits of the LQ and modulation of the exchange coupling at the qubit Rabi frequency performs $z$-rotations. Heisenberg exchange between the two physical qubits of the LQ perform an $x$-rotation, and two-qubit gates are performed via exchange coupling between qubits of different LQs. Using this scheme a controlled phase shift gate can be achieved with two exchange couplings and two $z$-rotations.

In this Letter we present an alternative scheme for exchange-interaction-only universal computation. Our scheme is similar to that of DiVincenzo et al [12] in that the LQ is encoded in the $S_{z}=+1 / 2$ subspace of a three qubit system. Single qubit rotations are then performed via the exchange coupling between physical qubits within the LQ and no $g$-factor engineering is required. In our scheme the LQ in its idle mode has equal and constant exchange couplings between the three physical qubits, analogous to previously proposed IFS architectures with always-on qubit couplings $[9,10$. To generate three equal couplings between the physical qubits, we propose ar- 


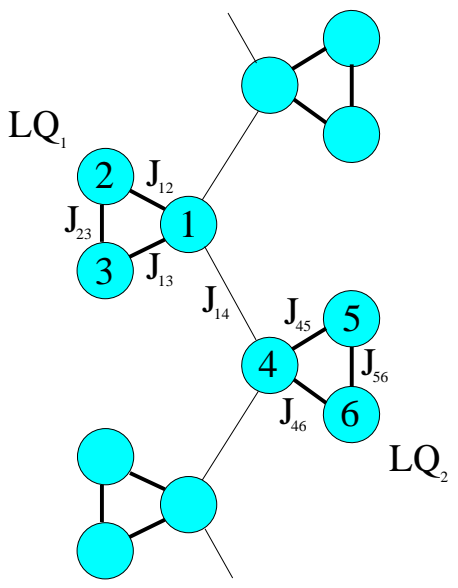

FIG. 1: (Color online) Suggested layout for encoded or logical qubits (LQ). Each LQ is composed of three physical qubits arranged in an equilateral triangle. The lines between physical qubits represent exchange interactions. The thick lines within LQs show always-on exchange interactions which are modified to perform single qubit rotations. The thin lines between LQs represent weaker exchange interactions which are turned on to perform two-qubit gates.

ranging them in equilateral triangles as shown in Fig. 1] In addition, the system is placed in a uniform magnetic field. The combination of the always-on couplings and the magnetic field creates an energy gap between the LQ subspace, which is the degenerate ground state, and the other states of the system. This gap makes the system more robust against decoherence 21]. Single qubit rotations are still performed by changing the strength of the exchange coupling between two physical qubits and can now be done both in the positive and negative sense. Two LQ operations, however, are simplified enormously due to the gap. With adiabatic modification of the interactions the system returns to the logical subspace when the couplings between LQs are turned off. Adiabatic evolution of interactions is already a requirement for all approaches using spins in quantum dots 22]. A conditional phase gate requires modification of only three exchange couplings all of which can be performed simultaneously.

We now analyze the dynamics of the above system and outline how to implement a universal quantum computation. The Heisenberg exchange interaction between two spins is

$$
H_{i j}=J_{i j} \mathbf{S}^{i} \cdot \mathbf{S}^{j}=J_{i j}\left(S_{x}^{i} S_{x}^{j}+S_{y}^{i} S_{y}^{j}+S_{z}^{i} S_{z}^{j}\right),
$$

where $\mathbf{S}=\sigma / 2$ and $J_{i j}$ is the coupling strength between spins $i$ and $j$. When placed in a uniform magnetic field there is an additional Zeeman interaction

$$
H_{z}=-h \sum_{i} S_{z}^{i}
$$

where $h=g B$ depends on the gyromagnetic ratio, $g$, which is the same for all dots, and the magnetic field strength, $B$. The Hamiltonian of a single LQ is thus

$$
H_{L Q}=H_{z}+H_{12}+H_{13}+H_{23} .
$$

In the LQ's idle mode, the coupling exchange between all pairs of physical qubits is equal (to 1 for simplicity) $J_{12}=J_{13}=J_{23}=1$. When $h$ is non-zero the system has a doubly degenerate ground state corresponding to the $S_{z}=+1 / 2$ subspace, as seen in Fig. 22 The orthogonal basis states of the LQ are chosen to span this space. In terms of the physical qubits the states are:

$$
\begin{aligned}
& \left|0_{L}\right\rangle=\frac{1}{\sqrt{2}}|\uparrow \uparrow \downarrow\rangle-\frac{1}{\sqrt{2}}|\uparrow \downarrow \uparrow\rangle \\
& \left|1_{L}\right\rangle=\frac{1}{\sqrt{6}}|\uparrow \uparrow \downarrow\rangle+\frac{1}{\sqrt{6}}|\uparrow \downarrow \uparrow\rangle-\frac{2}{\sqrt{6}}|\downarrow \uparrow \uparrow\rangle .
\end{aligned}
$$

With this choice, initialization can be done by breaking the degeneracy through raising or lowering one of the couplings and waiting for the system to decay to its ground state [12]. Also note that $\left|0_{L}\right\rangle$ incorporates the singlet state between spins 2 and 3 and $\left|1_{L}\right\rangle$ incorporates triplet states between the same spins. State $\left|0_{L}\right\rangle$ is antisymmetric with respect to exchange of sites 2 and 3 , while state $\left|1_{L}\right\rangle$ is symmetric for this exchange. This allows for readout of the LQs via singlet-triplet measurement schemes [16].

We choose the strength of the external magnetic field so as to maximize the energy gap between the doubly degenerate ground state and other states. The energy gap protects against many types of decoherence by forcing these processes to add energy to the system [21]. For the above encoding single-spin flips and arbitrary global magnetic fields cannot effect the LQs without forcing the system to leave the LQ subspace, requiring energy to overcome the gap. Single-spin phase operations, are not protected by the gap since they do not force the system out of the subspace and, thus, do not require energy. In general, the energy gap increases the robustness of the computation by inhibiting decohering processes which would cause the system to leave the subspace. The optimal external magnetic field to maximize the energy gap is $h=.75$ as shown in Fig. 2

Changing the strength of exchange couplings between physical qubits within the LQ, as in [12], performs single LQ rotations while always keeping the state of the system in the LQ subspace. Because the LQs have non-zero idle mode exchange couplings, positive and negative LQ rotations can be performed.

$$
H_{1}=\frac{1}{4}\left(\begin{array}{cc}
-J_{12}+2 J_{23}-J_{13} & \sqrt{3}\left(J_{12}-J_{13}\right) \\
\sqrt{3}\left(J_{12}-J_{13}\right) & J_{12}-2 J_{23}+J_{13}
\end{array}\right)
$$

is the LQ Hamiltonian for the general case written in the basis $\left[\left|0_{L}\right\rangle,\left|1_{L}\right\rangle\right]$. The Hamiltonian is defined within a constant times the identity which merely applies a global phase. From the general Hamiltonian we see that changing only $J_{23}$ performs a $z$-rotation and changing only $J_{12}$ 


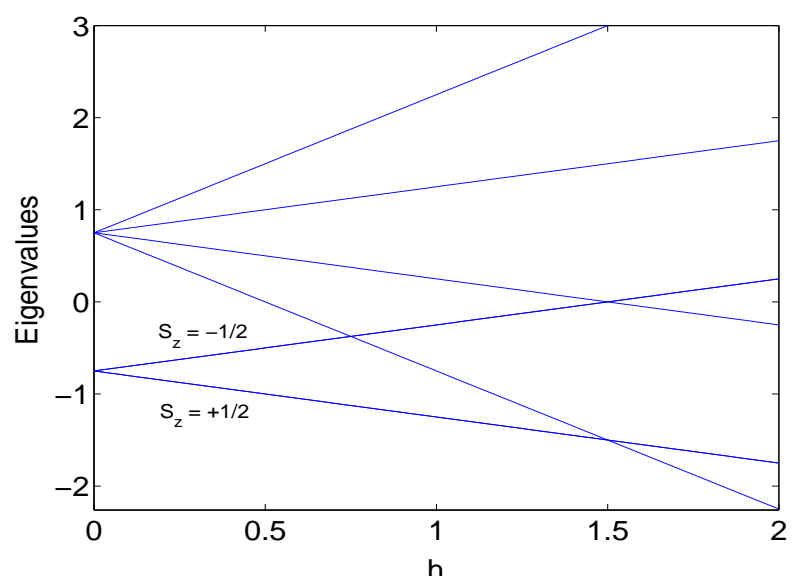

FIG. 2: (Color online) Eigenvalues of one LQ as a function of global magnetic field, $h=g B$. The eigenvalues marked $S_{z}=$ $+1 / 2$ and $S_{z}=-1 / 2$ are doubly degenerate. For suppression of decoherence, maximizing the gap between the $S_{z}=+1 / 2$, states used for the LQ and the remaining states is desirable. The gap is maximized for $h=.75$.

or $J_{13}$ performs a rotation about the axis in the $x-z$ plane $120^{\circ}$ from the $z$ axis. The eigenvalues of a LQ for these operations as a function of the change in coupling is shown in Fig. 3. Combinations of the above are sufficient to perform arbitrary $S U(2)$ rotations. We also note that it is possible to achieve $x$-rotations by changing $J_{12}$ and $J_{23}$ such that $\Delta J_{12}=2 \Delta J_{23}$.

There are numerous ways to couple LQs of the type described to allow universal quantum computation. Here, we describe one of the simplest approaches, a single exchange coupling between physical qubits 1 and 4 of Fig. [1. This coupling keeps the symmetry of the individual LQs, namely exchange of spins 2 and 3 . Thus, a $J_{14}$ couplings results in a diagonal inter-LQ interaction in the two-LQ basis $\left[\left|00_{L}\right\rangle,\left|01_{L}\right\rangle,\left|10_{L}\right\rangle,\left|11_{L}\right\rangle\right]$. We can write the effective Hamiltonian as

$$
H_{2}=\left(\begin{array}{cccc}
\lambda_{00} & 0 & 0 & 0 \\
0 & \lambda_{01} & 0 & 0 \\
0 & 0 & \lambda_{01} & 0 \\
0 & 0 & 0 & \lambda_{11}
\end{array}\right),
$$

where $\lambda_{10}=\lambda_{01}$. The eigenvalues as a function of $J_{14}$ are plotted in Fig. 4 along with the low-lying states outside of the LQ. The energy gap for leaving the logical subspace remains for all $J_{14}<0.75$. The eigenvalues themselves can be solved from the lowest solutions to the following equations:

$$
\begin{aligned}
0= & 4 \lambda_{00}-\left(J_{14}-3\right) \\
0= & 16 \lambda_{11}^{2}+8 J_{14} \lambda_{11}-3 J_{14}^{2}+16 J_{14}+27 \\
0= & 64 \lambda_{01}^{3}+16\left(J_{14}+9\right) \lambda_{01}^{2}-4\left(5 J_{14}^{2}-14 J_{14}+9\right) \lambda_{01} \\
& +3 J_{14}^{3}-23 J_{14}^{2}+37 J_{14}-81 .
\end{aligned}
$$

The solutions, particularly for $\lambda_{01}$, are rather involved and are omitted. The Taylor expansions for small $J_{14}$

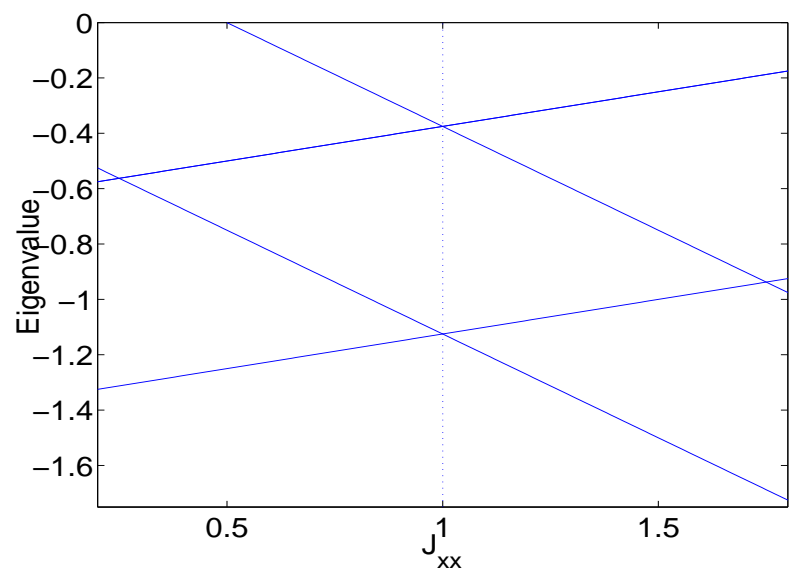

FIG. 3: (Color online) Eigenvalues as a function of coupling strength $J_{x x}=J_{12,13,23}$ during application of one-LQ gates. The idle-mode exchange coupling value, $J_{x x}=1$, is marked by the vertical dashed line. A $\sigma_{z}$ rotation is implemented by changing $J_{23}$ from its idle mode value. This breaks the degeneracy of the $\left|0_{L}\right\rangle,\left|1_{L}\right\rangle$ ground state but does not mix the states. Changing $J_{12}$ or $J_{13}$ implements a rotation around an axis in the $x-z$ plane $120^{\circ}$ away from the $z$-axis by breaking the doubly degenerate ground state into the states $\frac{\sqrt{3}}{2}\left|0_{L}\right\rangle-$ $\frac{1}{2}\left|1_{L}\right\rangle$ and $\frac{1}{2}\left|0_{L}\right\rangle+\frac{\sqrt{3}}{2}\left|1_{L}\right\rangle$. Combinations of the two rotations can implement arbitrary one-qubit rotations. The energy gap to leave the LQ subspace remains as long as the strength of the coupling being changed does not cause a level crossing; that is, $0.25<J_{x x}<1.75$.

are

$$
\begin{aligned}
& \lambda_{00}=-\frac{9}{4}+\frac{1}{4} J_{14} \\
& \lambda_{11}=-\frac{9}{4}-\frac{1}{12} J_{14}-\frac{4}{27} J_{14}^{2}-\frac{8}{243} J_{14}^{3}+\cdots \\
& \lambda_{01}=-\frac{9}{4}+\frac{1}{36} J_{14}-\frac{2}{27} J_{14}^{2}-\frac{110}{6561} J_{14}^{3}+\cdots
\end{aligned}
$$

When the interaction is modified adiabatically [22], the system is left in the logical subspace after $J_{14}$ is turned off. This is due to the energy gap generated by the always-on exchange interactions within the LQ. Removing leakage outside the logical subspace greatly simplifies the gate compared with the gate required in the case with no energy gap 12.

The two-LQ interaction plus equal $z$ rotations on each of the two LQs performs a conditional phase gate. Thus, we can implement a universal set of gates [23]: single qubit rotations, by changing the exchange couplings within a LQ, and conditional phase gates via the two LQ interaction, turning on the $J_{14}$ exchange coupling. We further note that the operations to perform the conditional phase gate may be done simultaneously, thus the gate requires only one time interval.

Up to this point we have not specified a physical system for our scheme. We now do so to insure that the strength of magnetic field required is reasonable. Of the 


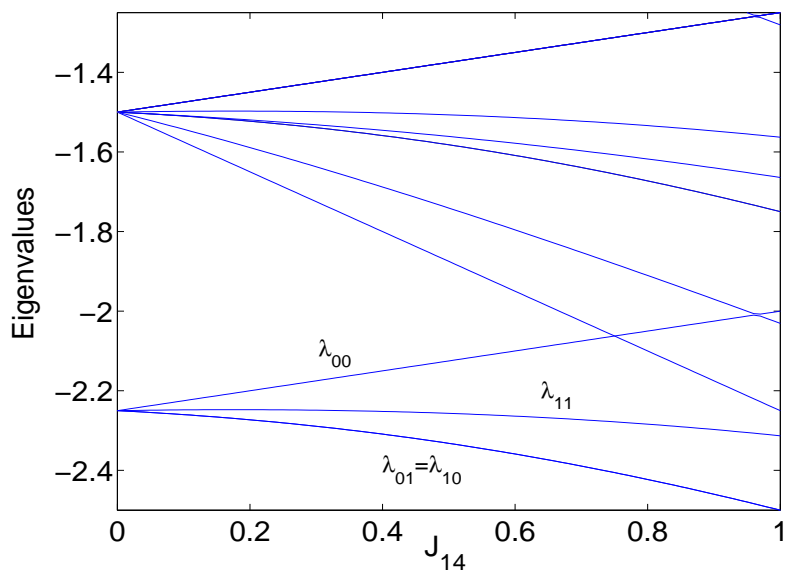

FIG. 4: (Color online) Eigenvalues of two LQs as a function of $J_{14}$. The coupling breaks the LQ's four-fold degenerate ground state into the states (from top to bottom) $\left|00_{L}\right\rangle,\left|11_{L}\right\rangle$, and the degenerate $\left|01_{L}\right\rangle$ and $\left|10_{L}\right\rangle$. The energy gap to leave the LQ subspace remains for all $J_{14}<.75$.

materials proposed for quantum computation with quantum dots [24, 25, 26, 27], GaAs has the $g$-factor with smallest magnitude, $g=-.44$. Approximating the required strength of the magnetic field using values found in Ref. 28], $J \sim 7 \mu \mathrm{eV}$ 29], the optimal value of $h=.75$ in units of $J$ can be achieved with magnetic field strength $B \sim .2 \mathrm{~T}$. For a LQ in the idle mode, this gives an energy gap between the LQ subspace and the rest of the states of $\Delta E \sim 5 \mu \mathrm{eV}$. In Si, which has a larger $g$-factor, the required magnetic field is even smaller.

In conclusion, we have demonstrated a scheme which suppresses decoherence via an energy gap between the logical qubit states and all other states and performs universal quantum computation using only the Heisenberg exchange process. The scheme calls for embedding each logical qubit in three physical qubits coupled by alwayson Heisenberg exchange interactions. Positive and negative single qubit rotations are simply implemented by changing exchange interactions between physical qubits within a logical qubit. Placing the system in a magnetic field opens an energy gap between the logical qubit subspace and all other states. The energy gap greatly simplifies the implementation of the two logical qubit gates protects against decoherence. Conditional phase gates can be implemented with just three exchange gates all of which can be done simultaneously. The conditional phase gate combined with the one logical qubit gates are sufficient for universal quantum computation.

[1] P. Zanardi, M. Rasetti, Phys. Rev. Lett., 79, 3306, (1997).

[2] L-M. Duan, G-C. Guo, Phys. Rev. Lett., 79, 1953,
(1997).

[3] E.M. Fortunato, L. Viola, J. Hodges, G. Teklemariam, D.G. Cory, New J. Phys. 4, 5, (2002).

[4] D. Kielpinski, V. Meyer, M.A. Rowe, C.A. Sackett, W.M. Itano, C. Monroe, D.J. Wineland, Science, 291, 1013, (2001).

[5] J.B. Altepeter, P.G. Hadley, S.M. Wendelken, A.J. Berglund, P.G. Kwiat, Phys. Rev. Lett., 92, 147901, (2004); P.G. Kwiat, A.J. Berglund, J.B. Altepeter, A.G. White, Science, 290, 498, (2000).

[6] X. Zhou, M. Wulf, Z. Zhou, G. Guo, M.J. Feldman, Phys. Rev. A, 69, 030301(R), (2004).

[7] M. Mohseni, J.S. Lundeen, K.J. Resch, A.M. Steinberg, Phys. Rev. Lett., 91, 187903, (2003).

[8] J.E. Ollerenshaw, D.A. Lidar, L.E. Kay, Phys. Rev. Lett., 91, 217904, (2003).

[9] X. Zhou, Z. Zhou, G. Guo, M.J. Feldman, Phys. Rev. Lett., 89, 197903, (2002).

[10] S.C. Benjamin, S. Bose, Phys. Rev. Lett., 90, 247901, (2003).

[11] Z. Zhou, B. Yu, X. Zhou, M.J. Feldman, G. Guo, Phys. Rev. Lett., 93, 010501, (2004).

[12] D.P. DiVincenzo, D. Bacon, J. Kempe, G. Burkard, K.B. Whaley, Nature (London), 408, 339, (2000).

[13] J. Levy, Phys. Rev. Lett., 89, 147902, (2002).

[14] L.-A. Wu, D.A. Lidar, M. Friesen, Phys. Rev. Lett., 93, 030501, (2004).

[15] D. Loss, D.P. DiVincenzo, Phys. Rev. A, 57, 120, (1998).

[16] B.E. Kane, Nature (London), 393, 133, (1998).

[17] R. Vrijen, E. Yablonovitch, K. Wang, H.W. Jiang, A. Balandin, V. Roychowdhury, T. Mor, D. DiVincenzo, Phys. Rev. A, 62, 012306, (2000).

[18] G. Burkard, D. Loss, D.P. DiVincenzo, Phys. Rev. B, 59, 2070, (1999).

[19] Y. Kato, R.C. Myers, D.C. Driscoll, A.C. Gossard, J. Levy, D.D. Awschalom, Science, 299, 1201, (2003).

[20] S.C. Benjamin, Phys. Rev. A, 64, 054303, (2001).

[21] D. Bacon, K.R. Brown, K.B. Whaley, Phys. Rev. Lett., 87, 247902, (2001).

[22] J. Schliemann, D. Loss, A.H. MacDonald, Phys. Rev. B, 63, 085311.

[23] A. Barenco, C.H. Bennett, R. Cleve, D.P. DiVincenzo, N. Margolus, P. Shor, T. Sleator, J.A. Smolin, H. Weinfurter, Phys. Rev. A, 52, 3457, (1995).

[24] J. Levy, Phys. Rev. A, 64, 052306, (2001).

[25] M. Friesen, P. Rugheimer, D.E. Savage, M.G. Lagally, D.W. van der Weide, R. Joynt, M.A. Eriksson, Phys. Rev. B, 67, 121301, (2003).

[26] N.J. Craig, J.M. Taylor, E.A. Lester, C.M. Marcus, M.P. Hanson, A.C. Gossard, Science 304, 565, (2004).

[27] J.M. Elzerman, R. Hanson, J.S. Greidanus, L.H. Willems van Beveren, S. De Franceschi, L.M.K. Vandersypen, S. Tarucha, L.P. Kouwenhoven, Phys. Rev. B 67, 161308, (2003).

[28] L. DiCarlo, H.J. Lynch, A.C. Johnson, L.I. Childress, K. Crockett, C.M. Marcus, M.P. Hanson, A.C. Gossard, Phys. Rev. Lett., 92, 226801, (2004).

[29] Ref. 28] provides experimental estimates for the singledot charging energy, $E_{C} \sim 500 \mu \mathrm{eV}$, and tunnel coupling, $t \sim 30 \mu \mathrm{eV}$. The coupling between dots is given by $J=$ $4 t^{2} / E_{C} \sim 7 \mu \mathrm{eV}$. 